\title{
Valerij Gal'cov
}

\section{Der Krieg und Ostpreußen im Gedächtnis der Einwohner des Gebietes Kaliningrad}

Verglichen mit dem gesamtrussischen Gedächtnis an den letzen Weltkrieg weist das lokale kollektive Gedächtnis der Einwohner des Gebietes [russ.: oblast'] Kaliningrad Besonderheiten auf. Es versteht sich, dass diese vor allem mit der Geschichte der Entstehung des Gebietes als Bestandteil der UdSSR verbunden sind. Gerade das Kriegsende in Ostpreußen und der Sieg über Deutschland waren ja die Ausgangspunkte für die Geschichte des Gebietes Kaliningrad. Mit anderen Worten, der Große Vaterländische Krieg mit Deutschland und der siegreiche Ausgang dieses Krieges sind nicht nur das Fundament der sowjetisch-russischen Präsenz in dieser Region, sondern auch die wichtigste ideologische Stütze der Staatsmacht und der Gesellschaft seit der Entstehung des Gebietes nach dem Krieg bis hinein in die Gegenwart. Diese grundlegenden Umstände bestimmen den Platz des Gedächtnisses an den Krieg und den Sieg, aber auch an Ostpreußen, im gesellschaftlichen Bewusstsein der Kaliningrader.

Das kollektive Gedächtnis bildet sich unter dem Einfluss vieler Faktoren heraus, jedoch liegen ihm bei alledem die Gesellschaft selbst, deren Unterscheidungsmerkmale und deren Geschichte zugrunde. Die Kaliningrader Gesellschaft hat sich nicht natürlich gebildet, sondern auf künstlichem Wege durch massenweise Umsiedlung von Menschen innerhalb kurzer Zeit und unter den extremen Bedingungen der Nachkriegszerstörungen.

Die vollständige Auswechslung der Bevölkerung auf dem Territorium des früheren Ostpreußen, das der Sowjetunion angegliedert wurde, erfolgte von 1946 bis 1948 mittels planmäßiger Massenzuwanderung von Sowjetmenschen anstelle der damals aus dem Gebiet nach Deutschland ausgesiedelten deutschen Staatsangehörigen (über 100000 ). Anfang der 1950er-Jahre, als der Prozess der Massenbesiedlung des Gebietes abgeschlossen war, lebten dort mehr als 500000 Menschen. Den Grundstock der Umsiedler bildeten Auswanderer aus Russland (70\%), Weißrussland (11\%), der Ukraine (7\%) und Litauen (4\%). Der Großteil von ihnen waren Bewohner jener Gebiete, die sich in den Kriegsjahren in der Besatzungszone befanden.

Im Wesentlichen waren dies elende, während des Krieges obdachlos gewordene, von Krieg und Zerstörung erschöpfte Menschen, die ihre Angehörigen und Freunde verloren hatten. Sie hofften darauf, die schwierige Nachkriegszeit zu überstehen, indem sie die Beihilfen der Regierung für Umsiedler in Anspruch nahmen. Aus diesem Grund ist es nicht verwunderlich, dass viele von ihnen ihren neuen Wohnort als nur zeitweilig betrachteten und das ehemalige Ostpreußen für sie noch lange Jahre fremd blieb. Daraus rührt eines der schwerwiegenden psychologischen Probleme der ersten Kaliningrader Generation - das Problem nicht einfach nur des Wiederaufbaus und der Umgestaltung der Wirtschaft des neuen 
Bodens, sondern auch die Schwierigkeit der Eingewöhnung, des sich Einlebens am neuen Ort.

Ihr Familiengedächtnis, das den wichtigsten Teil des kollektiven Gedächtnisses darstellt, hat Ereignisse der Kriegszeit bewahrt, die mit anderen Orten zu tun haben, die sich entweder in der Besatzungszone oder im Hinterland befanden. Gerade mit jenen konkreten Heimatorten war die Erinnerung an die umgekommenen oder verstümmelten Angehörigen und Freunde verbunden, an die Schrecken der Besatzung, an ein Leben in ständigem Hunger und an harte Arbeit. Zum Gedächtnis an den Krieg kamen die Eindrücke und später auch die Erinnerungen an das Leben auf dem neuen Boden. Dies alles entwickelte sich unter dem beträchtlichen Einfluss der offiziellen Propaganda, die zu Beginn vor allem die Teilnehmer der Kämpfe in Ostpreußen betraf, die weiterhin auf dem eroberten Boden lebten.

Im Jahr 1945 hatten der Hass auf den Feind und die Rachegefühle hier ihren Höhenpunkt erreicht. Gerade zu jenem Zeitpunkt wurde von der Kriegspropaganda ein bedrohliches Bild von Ostpreußen und dessen Bewohnern gezeichnet, das nach dem Krieg von den Massenmedien und in der propagandistischen Literatur verstärkt wurde: „Gewaltiger militärischer Brückenkopf“, „Höhle des Feindes“, „Herz des preußischen Militarismus“. In den Zeitungen hieß es: „Im Verlauf seiner jahrhundertelangen Geschichte war Preußen der Erzfeind der gesamten freiheitsliebenden Menschheit. Seine Hauptstadt Königsberg war eine Kriegsfabrik“, usw. Wie der über die Geschichte des Gebietes Kaliningrad forschende Historiker Jurij Kostjašov anmerkt, wurden vor diesem Hintergrund die „über Jahrhunderte entstandenen lokalen Traditionen und die auf ihrer Grundlage geschaffenen materiellen Werte nicht nur als minderwertig und wenig geeignet für Gebrauch und Reproduktion dargestellt, sondern auch als der bedingungslosen Zerstörung unterliegend, seien sie doch die Verkörperung all dessen, was in der Weltgeschichte am reaktionärsten und menschenfeindlichsten sei. In diesem Kontext wurde die völlige Zerstörung Ostpreußens (und der Tod seiner Einwohner) im Zuge der Kampfhandlungen als unzweifelhafter Segen ausgelegt. "1 Es ist nicht schwer, sich vorzustellen, wie sich die Beziehung der Sieger und ersten Umsiedler zu der ihnen feindlich erscheinenden Umwelt gestaltete. Die von der Staatsmacht aufgezwungene, vollkommene Loslösung vom Erbe praktisch der gesamten materiellen und geistigen Kultur Ostpreußens sowie die von der Bevölkerung im Großen und Ganzen unterstützte Idee der Schaffung eines wahrhaft sozialistischen, sowjetischen Gebietes an einem „sauberen Ort“ - all das führte einerseits zum gegenwärtigen armseligen Zustand der materiellen Überreste der früheren Zivilisation und bildete andererseits die Grundlange für die offizielle Version der Geschichte und des Bildes von Ostpreußen.

1 Jurij Kostjašov: Izgnanie prusskogo ducha: kak formirovalos' istoričeskoe soznanie naselenija Kaliningradskoj oblasti v poslevoennye gody. In: Jurij Kostjašov, Ėkchard Mattes (Eckhard Matthes): Izgnanie prusskogo ducha. Zapreščennoe vospominanie. Kaliningrad 2003, S. 14-15. 
Die siegreichen Ereignisse des Jahres 1945 waren Dreh- und Angelpunkt der militärisch-patriotischen Erziehung und der gesamten Arbeit des sowjetischen Propagandaapparats auf dem Territorium des Gebietes, wo die Propagandisten ihre Kräfte nicht für die Popularisierung der ruhmreichen Seiten der sowjetischen Vorkriegsvergangenheit zu verschwenden brauchten, weil es sie in dieser Region ganz einfach nicht gegeben hatte. Zudem kann man sagen, dass die Geschichte der ostpreußischen Kampfhandlungen von 1945 zusammen mit den Episoden des Siebenjährigen Krieges und den Schlachten der antinapoleonischen Koalition zu Beginn des 19. Jahrhunderts eines der wenigen Themen der Geschichte der Region war, die man in der Sowjetzeit mit Erlaubnis der Partei- und Staatsmacht erforschen und popularisieren konnte. Daher war die gesamte historische Heimatkunde im Gebiet Kaliningrad bis zum Ende des 20. Jahrhunderts im Grunde genommen eine militärgeschichtliche.

Die Kampagne zur militärisch-patriotischen Erziehung war auch deshalb ohne Beispiel, weil es im Gebiet eine beträchtliche Gruppierung der sowjetischen Armee und der Kriegsflotte gab. Hinzu kommt, dass ein Großteil dieser militärischen Einheiten 1945 an den Kriegsoperationen in Ostpreußen teilgenommen hatte und in ihnen natürlich die Erinnerung daran aufrechterhalten wurde. Die militärischen Kollektive selbst bildeten das Fundament der militärisch-patriotischen Erziehung.

In der Sowjetzeit war das Gedächtnis an den Krieg das markanteste Element des gesellschaftlichen Bewusstseins der Kaliningrader, wozu der äußerst militarisierte "Garnisonscharakter" der Region beitrug. Gleich nachdem die militärische Führung die Verwaltung des neuen Gebietes in die Hände der außerordentlichen zivilen Machtorgane gelegt hatte, gab der Leiter der Gebietsverwaltung für zivile Angelegenheiten, Vasilij Borisov, am 5. September 1946 den streng geheimen Erlass Nr. 131 „Über die verbotene Grenzzone auf dem Territorium des Gebietes Kaliningrad“ heraus, laut dem diese Zone „zum Zwecke der Verbesserung des Grenzschutzes" das gesamte Territorium des Gebietes umfassen sollte. De facto bedeutete dies eine beträchtliche Einschränkung der Bewegungsfreiheit und anderer Bürgerrechte der Kaliningrader und der Besucher des Gebietes. Auf der Grundlage dieses Befehls wurde im Gebiet im Laufe eines Monats eine durchgängige Passpflicht in die Tat umgesetzt, die sich übrigens auch auf die ländliche Bevölkerung erstreckte - etwas, was es damals in der Sowjetunion bekanntlich sonst nirgends gab. Unter den Bedingungen des beginnenden „Kalten Krieges“, des „eisernen Vorhangs" und der „Spionomanie“ wurde im Dezember 1950 sogar ein behördlicher Feiertag - der "Tag des Grenzsoldaten“ - durch eine Sonderverordnung des Gebietskomitees der KPdSU zu einem der wichtigsten Feiertage im Gebiet gemacht. ${ }^{2}$ In der Nach-Stalin-Ära, als das Grenzregime „milder“ wurde, blieben den Kaliningradern vier höchst geachtete, offizielle, jährliche Feiertage erhalten: der 9. April (Tag der Einnahme von Königsberg), der „Tag der Kriegsflotte“ (letzter Sonntag im Juli), der „Tag des Fischers“ (erster Sonntag im Juli) und natürlich der 
„Tag des Sieges“ am 9. Mai, der im postsowjetischen Russland zum „Feiertag der Feiertage" wurde, oder, wie der Schriftsteller Aleksandr Prochanov es ausdrückte, zum „Russischen Osterfest“.

Das strenge Sperr- und Grenzregime des Gebietes blieb bis Mitte der 1950erJahre bestehen, danach galt es für Sowjetbürger noch teilweise, für Ausländer jedoch war es in vollem Umfang bis1991 in Kraft. Nach dem Zerfall der UdSSR ergab sich für das Gebiet, das durch zwei Staatsgrenzen von Russland abgeschnitten war, erneut ein Sonderstatus. Ab Mai 2004, als sich das Gebiet innerhalb der Europäischen Union wiederfand, senkte sich abermals ein Vorhang herab, der „samtene“, wie er heute manchmal genannt wird. Der Sonderstatus des Gebietes übte einen wesentlichen Einfluss auf das gesellschaftliche Bewusstsein seiner Bewohner und ihr kollektives historisches Gedächtnis aus und wird dies auch in Zukunft tun.

In den 1960er und 1970er-Jahren entstand im Gebiet ein System der patriotischen Erziehung mittels eines Netzwerks historisch-heimatkundlicher Museen und Recherchegruppen auf schulischer Basis. Bis zur Perestroika gab es 104 Ruhmesmuseen in Schulen, in denen in der Regel Material zu den an den ostpreußischen Kriegsoperationen beteiligten militärischen Verbänden zusammengetragen wurde. Das indirekte Gedächtnis an den Krieg fand seinen Niederschlag auch in den damals populären wehrsportlichen Spielen für Kinder „Zarnica“ und „Orlenok“, die meist von Kriegsteilnehmern geleitet wurden. Insgesamt unterschied sich das kollektive Gedächtnis an den Krieg in Kaliningrad typologisch nicht vom offiziellen gesamtsowjetischen Bild des Krieges, außer durch dessen grundlegende Bedeutung für die gesamte Geschichte des Gebietes. Während der Perestroika und in den ersten postsowjetischen Jahren begann sich die Lage jedoch zu ändern.

In der Sowjetzeit bewegte sich die Selbstdefinition der Kaliningrader hauptsächlich im Rahmen ihrer Identifikation mit der Natur des Gebietes sowie mit der sowjetischen Geschichte und Kultur. Ende der 80er/Anfang der 90er-Jahre brach der Totalitarismus zusammen, der „eiserne Vorhang“ fiel, die Nachbarn Polen, Deutschland und andere Länder wurden frei zugänglich, es eröffneten sich große Möglichkeiten, alles über die Geschichte der Region zu erfahren, die alten Häuser der Kaliningrader wurden von ihren ehemaligen Besitzern besucht. Unter dem Einfluss dieser und anderer Faktoren keimten im Bewusstsein eines Teils der Gesellschaft neue Empfindungen und Ideen auf. Es begann ein langwieriger und mühsamer Prozess, im Laufe dessen man sich seiner Teilhabe am kulturhistorischen Erbe der Vorkriegszeit und seiner Mitwirkung am Schicksal der von diesem Boden vertriebenen Menschen bewusst wurde. Dabei muss berücksichtigt werden, dass es bereits drei Generationen gebürtiger Kaliningrader gibt und dass von den ca. 940000 hier lebenden Menschen mehr als 600000 im Gebiet Kaliningrad zur Welt gekommen sind.

Lange Zeit war es schwierig, sich ein aussagekräftiges Bild vom Alltag der Umsiedler zu verschaffen, da ein Großteil der Archivdokumente unter Verschluss gehalten wurde. Aber selbst als sie frei zugänglich wurden, stellte sich heraus, dass 
sich ohne „lebendige“ Zeugnisse der ersten Zuwanderer deren Nachkriegsleben auf dem neuen Boden einseitig-offiziös ausnehmen würde. Eine systematische Aufzeichnung dieser Zeugnisse wurde unter den Bedingungen von Glasnost und Perestroika in den Jahren von 1988 bis 1991 von Studenten und Dozenten der historischen Fakultät der Universität Kaliningrad unter der Leitung von Professor Kostjašov vorgenommen. Die veröffentlichten Erinnerungen der Umsiedler waren nicht nur eine einzigartige Auskunftsquelle über die Geschichte des Nachkriegsalltags im Gebiet Kaliningrad, sondern lieferten auch wertvolles Material, um die Evolution in der Einstellung der Zuwanderer zu den Problemen zu erforschen, mit denen sie sich zu Beginn ihres neuen Lebens auseinanderzusetzen hatten und für die sie eine Lösung finden mussten. ${ }^{3}$

Hier einige wenige Betrachtungen von Veteranen aus dem Gebiet: Jekaterina Koževnikova (geb. 1924) erinnert sich: „Es gab keine Wut auf die Deutschen. Es war uns irgendwie schwer ums Herz für dieses Volk. [...] Wir empfanden keinen Hass ihnen gegenüber. Nur ein Gefühl des Mitleids, dass es ihnen auch schwer gefallen sein muss, das zu verlassen, was sie sich erworben hatten, den Boden, auf dem sie lebten. Natürlich, ihre Kultur ist bewundernswert. [...] Diese Kultur hätte man nicht zerstören, sondern sie erhalten und zur Erziehung der Jugend nutzen sollen. Ich denke, die Deutschen müssen hierherfahren und wir dorthin, auch unsere Kinder und Enkel. Sie lieben die Arbeit und die Sauberkeit - ein prächtiges Volk, alle Achtung! Dass fanatische Führer geboren werden - die können in jedem Volk geboren werden, nicht nur bei den Deutschen. Ich möchte nicht alles ausradieren, wie das momentan viele Leute machen. Wir hatten viele dunkle Flecken auf unserer Geschichte, aber es gab auch Gutes. Auch wenn wir bescheiden lebten, so haben wir doch gedacht, dass es uns gut geht, wir haben nicht umsonst gelebt." (S.307)

Aleksandr Furmanov (geb. 1926) hat an den Kämpfen in Ostpreußen teilgenommen: „Damals habe ich gedacht, das die Deutschen zu Recht ausgesiedelt werden: Sie hatten ausgekämpft und dieser Boden gehörte rechtmäßig uns. Jetzt aber beginne ich zu verstehen, dass wir sündhaft einfach all das zerstört haben, was die Deutschen zurückgelassen haben, auch das Gute. Das war natürlich barbarisch. Aber man muss auch uns verstehen. In jenen Jahren war alles hier fremd, war deutsch. Und alle strebten danach, Faschismus und Preußentum für alle Zeiten auszurotten." (S.305)

Anna Bojko (geb. 1924): „In der ersten Zeit war unsere Beziehung zum neuen Territorium wie zu fremder Erde. Aber der Mensch gewöhnt sich an alles. Wir haben hier gleiche Häuser gebaut, anders als die deutschen Gebäude, die sich voneinander unterschieden. Wir haben unsere Kultur hierhergebracht und die Kultur der wahren Eigentümer vollständig zerstört. Und wir haben begonnen, unser sowjetisches Leben zu leben. Jetzt ist es schwer, über Fehler zu reden, die gemacht

3 Svetlana P. Gal' cova et al. (Hrsg.): Vostočnaja Prussija glazami sovetskich pereselencev: Pervye gody Kaliningradskoj oblasti v vospominanijach i dokumentach. 2. izd., ispr. i dop. Kaliningrad 2003. 
wurden. Das war eine andere Zeit, unsere Einstellung zu allem war eine völlig andere. [...] Damals schien uns, dass wir alles richtig machen. Aber wie auch immer - welchen Sinn hat es, über die Fehler zu reden, die gemacht wurden? Jetzt müssen wir versuchen, das zu retten, was noch in unseren Kräften steht. Und außerdem - nachdem ich mein ganzes Leben im Gebiet Kaliningrad verbracht habe, kann ich es nicht anders betrachten, als als meine Heimat. Diese Erde hat das Recht, böse auf uns zu sein, aber mir scheint, dass wir ihr trotz alledem lieb und teuer geworden sind.“(308)

In den letzten Jahren haben sich neue Töne in die Wahrnehmung des Krieges und des Sieges gemischt, vorwiegend bei den jungen Kaliningradern. Die Diskussionen über das Thema Gewalt und die von den Rotarmisten gegen die Zivilbevölkerung in Ostpreußen verübten faktischen Verbrechen werden hauptsächlich im russischsprachigen Internet geführt. Es gibt Teams, die aktiv nach sterblichen Überresten von Teilnehmern der Gefechte und von Kriegsopfern suchen. In Kaliningrad und im Gebiet Kaliningrad wurden einige neue deutsche und internationale Friedhöfe eröffnet. Man kann sagen, dass sich das Motiv der Aussöhnung im allgemeinen Chor der Verherrlichung des Sieges von 1945 immer lauter bemerkbar macht.

Eine nicht unwesentliche Rolle spielt dabei das Verhältnis zum früheren Ostpreußen. Derzeit kann diese Frage als aktuell angesehen werden, da die Antwort darauf direkt mit dem weiterhin andauernden Prozess der Selbstdefinition der Kaliningrader verbunden ist. Allein die Tatsache, dass das Gebiet Kaliningrad auf einem Teil des Territoriums von Ostpreußen geschaffen wurde und die für die Bevölkerung offiziell verbotene vorsowjetische Geschichte der Region machten das Thema „Ostpreußen“ marginal und geheimnisvoll. Daher wurde es mit der Zeit (seit Ende der 80er-Jahre) zwangsläufig zum Gegenstand erhöhter und wachsender Aufmerksamkeit. Mehr noch, sobald im Land Meinungs- und Redefreiheit aufkamen, begannen dahingegangene Erscheinungen des menschlichen Lebens wie „Ostpreußen“ und „Königsberg“ nicht einfach nur Beachtung zu finden, sondern sich bis zu einem gewissen Grad auf das Leben im Gebiet Kaliningrad auszuwirken.

Das neue sowjetische Gebiet war nach dem Krieg ein Territorium der besonders aktiven antideutschen (antiwestlichen, antikapitalistischen) Propaganda, die sich de facto in der praktisch vollständigen Ablehnung alles Deutschen sowohl auf verbaler als auch auf materieller Ebene manifestierte. Seit Beginn der 90erJahre fand keine gezielte Zerstörung der Überbleibsel der deutschen Zivilisation aus ideologischen Gründen mehr statt (teilweise auch deshalb, weil es kaum noch etwas zu zerstören gab), aber das heißt nicht, dass auch der Kampf der Ideen rund um die deutsch-preußische Vergangenheit ein Ende gefunden hätte. Im Gegenteil, dieser Kampf hat genau damals, mit der aufkommenden Gedankenfreiheit erst richtig begonnen und dauert bis heute an, wobei er manchmal abklingt und von Zeit zu Zeit wieder aufflammt. Die antikapitalistische Propaganda und die von den Machtorganen deklarierte „ideologische Geschlossenheit der Gesellschaft" sind Dinge der Vergangenheit; was geblieben ist, ist die ideologische Spal- 
tung der Kaliningrader Gesellschaft, bei der das Verhältnis zur deutschen Vergangenheit der Region und zu den Überresten der deutschen materiellen Kultur einen zentralen Platz einnimmt. Einerseits spielen sie eine wichtige Rolle für diejenigen, die der Ansicht sind, dass sich im Gebiet Kaliningrad eine spezielle ethnische Gemeinschaft herausbilde. Andererseits stellen sie eine Gefahr für die Bewahrer der traditionellen russischen Kulturwerte dar, also für jene, die der Überzeugung sind, dass nur sie echte russische Patrioten sind.

Die Kaliningrader Patrioten unterscheiden sich von ihren Gesinnungsgenossen in anderen Regionen dadurch, dass sie sich an „vorderster Front des Kampfes um die Seelen der Menschen “ empfinden. Sie teilen sie in „Eigene“ und „Fremde“ ein und fürchten nicht ohne Grund den (ihrer Meinung nach zersetzenden) Einfluss der Geschichte Ostpreußens und der Überbleibsel der deutschen Zivilisation auf die Kaliningrader. Dieser Einfluss stellt nach den Grundsätzen der traditionellen russischen-orthodoxen Werte die größte Gefahr für die Selbstdefinition der Kaliningrader dar; für diejenigen, die sich russische Patrioten nennen, kann von einer speziellen „Kaliningrader Identität“ keine Rede sein und schon gar nicht von der Identität einer „europreußischen“ oder Kaliningrader „Ethnie“.

Die „Patrioten“ haben hier in vielem recht - die Überlegungen hinsichtlich einer Kaliningrader „Ethnie“ sind meiner Ansicht nach naiv. Vielmehr kann man vorsichtig von einer speziellen Kaliningrader Identität sprechen - die Grundlagen und wesentlichen Kennzeichen der Mentalität der heutigen Kaliningrader, auch der jungen Leute, sind dieselben wie bei der Bevölkerung im übrigen Russland. Doch gibt es dabei natürlich auch Besonderheiten, die mit der Enklave-Situation und mit dem ostpreußischen Erbe zu tun haben. Eine andere Frage ist, inwieweit diese Besonderheiten die lokale Identität beeinflussen. Hierzu haben in Kaliningrad in erster Linie die Mitarbeiter der Baltischen Föderalen Immanuel-KantUniversität bereits etliche Arbeiten publiziert, in denen das Thema aus Sicht der Kulturwissenschaft, der Soziologie, der Sozialphilosophie, der Geschichtswissenschaft, der Psychologie usw. untersucht wird.

Hier einige Ergebnisse dieser Forschungen: Von 1991 bis 2000 hat die Fakultät für slawische Philologie und Journalistik im Gebiet volkskundliche Expeditionen mit dem Ziel durchgeführt, festzustellen ,auf welche Weise sich eine neue Kultur in der Region Kaliningrad entwickelt, welcher Faktor für die Herausbildung neuer kultureller Klischees am wichtigsten ist, ob ein Nivellierungsprozess der ethnischen Kulturen stattfindet und wenn ja, wie er verläuft". Besonderes Interesse im Hinblick auf die Entwicklung eines eigenen folkloristischen Milieus erweckten bei den Teilnehmern der Expedition die (vor relativ kurzer Zeit) entstandenen volkstümlichen Erzählungen, welche die Vorstellung von der Geschichte Ostpreußens reflektieren. „In ihnen vollzieht sich eine Rekonstruktion der deutschen mythologischen Vorstellungen von Bewohnern der Unterwelt (Gnomen bzw. Barstucken), Zwergen, dem deutschen Gespenst, den Geheimnissen von Königsberg, dem Geist der Königin Luise und unterirdischen Gängen und Schätzen. Mythische Motive wie jenes von den vier noch von den Preußen gepflanzten Eichen oder der Entstehung einzelner Festungen und Flüsse werden wiederbelebt oder 
neu geschaffen. Die Stadtgeschichte selber wird von den volkstümlichen Erzählungen als großes Geheimnis betrachtet, das sich in rätselhaften Gebäuden (Dom Sovetov), alten und neuen Brücken, Befestigungsanlagen und in der Errichtung oder der Zerstörung von Kirchen offenbart" (in Texten über die Demolierung der deutschen Kirchen stößt man häufig auf das Motiv der Verfluchung, mit dem „Zuweilen die Gründe für das schlechte Leben der Umsiedler im Gebiet Kaliningrad in der heutigen Zeit erklärt werden“). ${ }^{4}$ Die Ergebnisse, zu denen die Forscher gelangen, lassen sich wie folgt zusammenfassen: „Die multinationale $\mathrm{Zu}$ sammensetzung des Gebietes und eine Reihe von historischen, politischen und geographischen Ursachen, die die Entwicklung des kulturellen Milieus der Region beeinflusst haben, legen Zeugnis davon ab, dass das Gebiet in ethnischer und kultureller Hinsicht ein einzigartiges Mosaik aus Volkstraditionen darstellt. “5 „Auf dem Territorium des Gebietes Kaliningrad können wir in der Tat die Entstehung einer neuen folkloristischen Subkultur beobachten, in der sich Elemente der slawischen, baltischen und deutschen Kultur organisch miteinander verbunden haben, "6 wobei diese allerdings auf dem genannten Territorium noch keine nachhaltige Existenz und keinerlei strenge Genreformen angenommen haben.

Soziologen und Kulturwissenschaftler beobachten eine für die Kaliningrader Mentalität charakteristische Grundeigenschaft - eine stark ausgeprägte Paradoxie, deren merkwürdiger Kern „der ständige Vergleich/die ständige Gegenüberstellung/Verbindung von Altem und Neuem, Deutschem und Russischem, Vorkriegszeit und Nachkriegszeit, Eigenem und Fremden ist. Zwischen ihnen hat sich eine versteckte Konfrontation/Rivalität bewahrt, aber auch das Bestreben, Verbindung mit der Vergangenheit zu halten, auf deren langes Zurückreichen und historischen Stellenwert man stolz ist. Es gibt den Wunsch, dies als „Eigenes“ zu begreifen. Das wichtigste Element dieser Geisteshaltung - ihr unabänderliches Merkmal - ist das Gedächtnis an den Zweiten Weltkrieg als Ursache für die unmittelbare Existenz des Gebietes Kaliningrad und all seiner Besonderheiten. ${ }^{7}$

Der Krieg hat die Geschichte des Bodens jäh abreißen lassen, der heute „Gebiet Kaliningrad“ heißt und ihn unter dem Druck der staatlichen Organe für lange Jahre zu einem Boden ohne Vergangenheit gemacht. Daher spielt die Antwort auf die Frage, wie eine Beziehung zur fremden Vergangenheit aufgebaut werden kann, im Prozess der Selbstdefinition eine wichtige Rolle. Die Existenz dieses Problems hat das mit Traumata behaftete historische Gedächtnis der Kaliningrader Gesellschaft geformt. Die Versuche, die Vorkriegsvergangenheit der Region kennenzulernen und zu verstehen, um sie bis zu einem gewissen Grad zu seiner eigenen zu

4 Larisa Rubcova: Mežetničeskie otnošenija v russkom fol'klore Kaliningradskoj oblasti. In: Andrej P. Klemešev (red.): Na perekrestke kul'tur: russkie v Baltijskom regione. Vyp. 7, Č. 1. Kaliningrad 2004, S. 200, 201.

5 E.A. Galkina: Fol'klor v kontekste regional'nych cennostej i svjazej. In: Vestnik Baltijskogo naučnogo centra 1996. Nr. 1 (6), S. 67.

6 Rubcova, Mežètničeskie otnošenija, S. 202, 203.

7 Larisa Gavrilina: Kaliningradskij tekst kak metatekst kul'tury. In: Kantovskij sbornik 2010. Nr. 3 (33), S. 73-74. 
machen bzw. in dieser fremden Vergangenheit Fragmente seiner eigenen, vertrauten Geschichte zu finden, haben das Dilemma zwischen dem „Eigenen“ und dem „Fremden ““ in den Vordergrund gerückt.

In der Praxis werden die Bilder von Königsberg und Ostpreußen von den verschiedenen Schichten der Kaliningrader Gesellschaft unterschiedlich genutzt. Für die staatlichen und politischen Entscheidungsträger werden diese Phantome manchmal zu so etwas wie einer "Spielfigur“ in der Politik; dies ist ein wichtiger Faktor in der ideologischen und politischen Auseinandersetzung in der Region (rund um die vermeintliche „Germanisierung“ des Gebietes, im Kontext der Beziehungen Russlands zu Europa, „Eigenem“ und „Fremdem“, des Schutzes der russischen Kultur und der „Entlarvung“ ihrer „Verräter“, usw.). Für die Kaliningrader Kulturschaffenden (insbesondere Schriftsteller, Maler und Dichter) ist das eine ihrer Inspirationsquellen. Unter den Bedingungen der völligen Kommerzialisierung des Lebens (bei vielen auch der Seelen) der Menschen und der Herrschaft der internationalen Massenkultur ist „Ostpreußen“ eine attraktive, gewinnbringende und für russische Verhältnisse exklusive „Marke“: In Kaliningrad und im Gebiet Kaliningrad gibt es Dutzende Handelsmarken, Organisationen und Firmen, in deren Bezeichnung ostpreußische Ortsnamen oder historisch-kulturelle Symbole vorkommen; gerade in Bezug auf Marken und Konsum werden Königsberg und Ostpreußen von den Kaliningradern in erster Linie wahrgenommen. Dies muss bei der Analyse der Besonderheiten der lokalen Identität berücksichtigt werden, ohne die Bedeutung dieser Besonderheiten für den schwierigen Prozess der Selbstdefinition zu übertreiben. Zu guter Letzt ist „Ostpreußen“ für Geistesarbeiter ein vielgestaltiges, komplexes, interessantes und langfristiges Forschungsobjekt, wofür unter anderem der vorliegende Artikel als Beleg dienen kann. 
